\title{
Thick-film permanent magnets by membrane electrodeposition
}

\author{
F. M. F. Rhen, ${ }^{\text {a) }}$ E. Backen, and J. M. D. Coey \\ Physics Department, Trinity College, Dublin 2, Ireland
}

(Received 20 December 2004; accepted 29 March 2005; published online 3 June 2005)

\begin{abstract}
Thick films of nearly equiatomic Co-Pt produced by electrodeposition into nanoporous membranes exhibit an isotropic magnetic phase with square hysteresis loops $\left(M_{r} / M_{s}=0.95\right)$ and coercivity of up to $1.3 \mathrm{~T}$. The initial magnetization curve is characteristic of pinning-type magnets. The coercivity can be controlled by the choice of annealing temperature or alloy composition, which depends on the chemical composition of the plating bath, properties which can be exploited to produce fine-pitch pole patterns. (C) 2005 American Institute of Physics. [DOI: 10.1063/1.1923587]
\end{abstract}

\section{INTRODUCTION}

There is a growing interest in using magnetic components in microelectromechanical systems (MEMS). When all the dimensions of a magnetic system are shrunk by a factor $k$, the magnetic force resulting from the interaction between two magnets is enhanced by a factor $k$, whereas the force for a magnet-coil interaction is scale invariant. Magneticmoment density $M$ and current density $j$ are assumed to be constant. The scaling of the magnetic forces can be explained as follows. The magnetic dipole field (stray field) of a magnet $B \sim \mu_{0} m r^{-3}$ is scale invariant-the magnetic moment $m$ of an element of magnetization $\mathbf{M}$ scales as $k^{-3}$, whereas the magnetic field $\mathbf{B}$ falls off as $r^{-3}$, which scales as $k^{3}$. However, the magnetic moment of a coil, $m_{\text {coil }} \sim \mathrm{I} A \sim j a A$ where $a$ is the conductor cross section and $A$ is the coil area, scales as $k^{-4}$, hence the magnetic field scales as $k^{-1}$. The force on another magnet is proportional to $\nabla \mathbf{B}$, hence the force between the two small dipoles scales as $k$, whereas the force between a dipole and a coil is scale invariant. It is therefore more favorable to use permanent magnets to exert forces in microscale mechanical devices than it is to use coils. ${ }^{1,2}$ Furthermore, coils cannot compete with magnets for producing steady fields when dimensions are less than about a millimeter because of the difficulties of accommodating the necessary number of ampere turns, and dissipating the heat generated by current densities $j \sim 10^{9} \mathrm{~A} \mathrm{~m}^{-2}$.

In order to produce magnetic MEMS devices, we need thick films which can be magnetized in pole patterns which vary on a scale comparable to the film thickness. Pole patterns are required for micromotors, which are a basic constituent of many MEMS devices such as variable capacitors and inductors. Permanent magnets are only useful because of the stray field they produce. A uniformly magnetized film produces no stray field, except around its edges. The future of magnetic MEMS will be conditioned by our ability to produce thick $(100-500 \mu \mathrm{m})$ permanently magnetized films, and to pattern them magnetically on a similar scale.

Thick films of high-performance permanent magnets based on $\mathrm{Sm}-\mathrm{Co}$ or $\mathrm{Nd}-\mathrm{Fe}-\mathrm{B}$ alloys can be produced by fast sputtering or screen printing techniques using coercive pow-

\footnotetext{
a) Author to whom correspondence should be addressed; FAX: +353-16711759; electronic mail: rhenf@tcd.ie
}

der. The sputtered films require subsequent heat treatment to develop the necessary phase microstructure. ${ }^{3-5}$ Subsequent imposition of a desired pole pattern requires local application of a large magnetic field. Traditionally this is achieved using a high current pulse in a suitably designed magnetizing fixture, but we have seen that it is difficult to miniaturize the coils beyond a certain point, as the field produced by a conductor with a given limiting current density scales as $1 / k$.

Here we describe a method of preparing small permanent magnet structures by electrodeposition into a membrane template. Nanoporous membranes are obtained by anodizing aluminum $^{6}$ or by selective dissolution of self-organized block copolymers. ${ }^{7}$ There are no procedures for preparing rare-earth alloys by electrodeposition, but coercive thin films can be made from $\mathrm{Co}-\mathrm{Pt},{ }^{8,9} \mathrm{Co}-\mathrm{Pd},{ }^{10}$ and $\mathrm{Fe}-\mathrm{Pt} .{ }^{11}$ For $\mathrm{Co}-\mathrm{Pt}$, there are two useful composition ranges, one is near the equiatomic $\mathrm{Co}_{50} \mathrm{Pt}_{50}$ composition where the ordered $L 1_{0}$ phase is stable below about $700{ }^{\circ} \mathrm{C}$ but usually has to be produced by annealing a disordered fcc CoPt alloy; the other is cobalt rich near $\mathrm{Co}_{80} \mathrm{Pt}_{20}$. The latter, which typically includes small amounts of other atoms such as $\mathrm{P}, \mathrm{Ta}$, and $\mathrm{Cr}$, is widely used in thin-film magnetic recording media. Coercive films can be obtained by electrodeposition in this composition range, without any need for annealing. ${ }^{12}$

There are recent reports of electrodeposition of ferromagnetic metals and alloys into nanoporous membranes. ${ }^{13-15}$ Some coercivity $(0.16 \mathrm{~T})$ has been reported for cobalt deposited into alumina templates, and there are recent reports of Co-Pt nanotubes ${ }^{16}$ and nanowires ${ }^{17,18}$ with interesting hysteretic properties prepared in this way.

\section{EXPERIMENT}

Here we use commercial alumina membranes with a 200-nm pore size from Whatman. The pores constitute about $50 \%$ of the membrane volume. Membrane thickness is $66 \mu \mathrm{m}$. A back electrode of 100-200 nm of Pt or Ag was produced by sputtering, and the pores were filled with equiatomic CoPt from a bath which includes both ions in solution. ${ }^{19}$ The electrochemical bath contains $0.1 \mathrm{M}$ of cobalt sulfamate, $15-35 \mathrm{mM}$ of dinitrodiamimine platinum salt, also known as platinum p-salt, and $0.1 \mathrm{M}$ of diammonium hydrogen citrate. Solution $\mathrm{pH}$ was adjusted to 5 by adding $\mathrm{NaOH}$ and the electrodeposition was carried out at room 


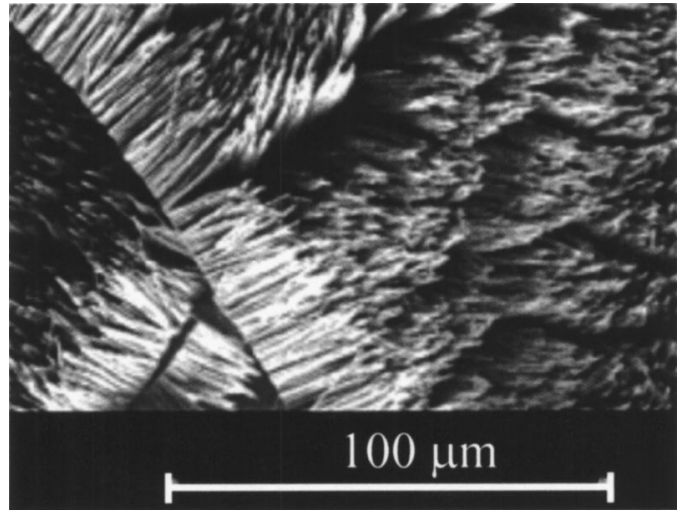

FIG. 1. Micrograph of electrodeposited CoPt nanowires after dissolution of the membrane template.

temperature. The bath containing a high concentration (35 $\mathrm{mM}$ ) of dinitrodiammine platinum provided a deposition rate which of about $6-8 \mu \mathrm{m} \mathrm{h}^{-1}$, which is higher than that obtained in a conventional sputtering process. Plating was carried out at room temperature in open atmosphere. No stirring is used during the deposition and the potential was varied in the range -2.5 to $-5.0 \mathrm{~V}$ vs $\mathrm{Ag} / \mathrm{AgCl}$. Deposits carried out galvanostatically at $25 \mathrm{~mA} \mathrm{~cm}^{-2}(-5 \mathrm{~V}$ vs $\mathrm{Ag} / \mathrm{AgCl})$ were annealed in vacuum of $10^{-6}$ bar or in hydrogen. First, a furnace was heated up to the desired temperature, and then the sample under vacuum was pushed into the furnace and after the annealing quenched in water. The stray field was measured using an $x-y-z$ stage with a small Hall probe giving a spatial resolution of about $250 \mu \mathrm{m}$. Magnetization measurements were carried out in a 5-T superconducting quantum interference device (SQUID) magnetometer from Quantum Design. X-ray diffraction was carried out using $\mathrm{Cu} K \alpha$ radiation. The membrane template was dissolved in $3 \mathrm{M}$ of $\mathrm{NaOH}$ solution for the samples used for scanning electron microscopy, all the other measurements were carried with the membrane intact.

\section{RESULTS AND DISCUSSION}

The composition of the deposits was found to be almost independent of the potential/current density in the range

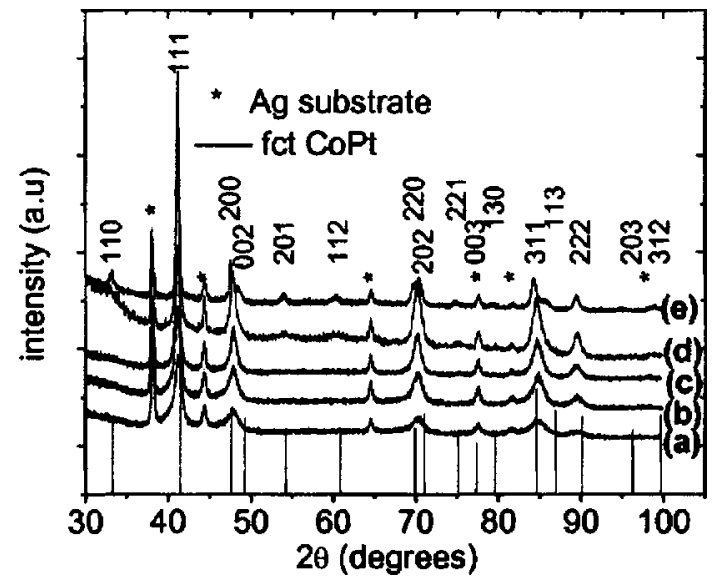

FIG. 2. X-ray diffraction patterns of $\mathrm{Co}_{50} \mathrm{Pt}_{50}$ deposited in a 200-nm alumina membrane. (a) As-deposited and annealed at (b) 450, (c) 550, (d) 650, and (e) $700{ }^{\circ} \mathrm{C}$ for $30 \mathrm{~min}$.

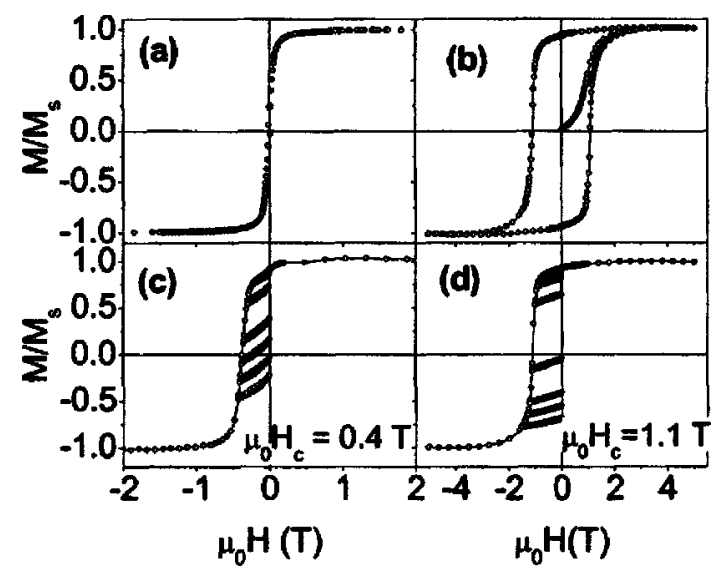

FIG. 3. Room-temperature magnetization curves of (a) as-deposited CoPt, (b) films annealed at $700{ }^{\circ} \mathrm{C}$ for $30 \mathrm{~min}$ and room-temperature recoil curves of CoPt annealed at (c) 650 and (d) $700{ }^{\circ} \mathrm{C}$.

studied $-2.5 \mathrm{~V}\left(10.2 \mathrm{~mA} \mathrm{~cm}^{-2}\right)$ to $-5 \mathrm{~V}\left(25 \mathrm{~mA} \mathrm{~cm}^{-2}\right)$ vs $\mathrm{Ag} / \mathrm{AgCl}$. Due to the cylindrical shape of the electrochemical cell with the membrane in at the bottom, the reference electrode could not be placed near the membrane, which accounts for the high potential measured. Figure 1 shows a top view of the electrodeposited $\mathrm{CoPt}$ wires after the dissolution of the 200-nm membrane. The length of the wires is about $30 \mu \mathrm{m}$.

As-deposited films were magnetically soft, with a small grain size, and a disordered fcc structure (Fig. 2). As the annealing temperature is increased, the disordered phase is progressively converted to the $L 1_{0}$-ordered phase. At $700{ }^{\circ} \mathrm{C}$ the main phase present is fct $\mathrm{CoPt}$ (Fig. 2), which shows high coercivity as presented in Fig. 3(b). The coercivity reaches a maximum of $1.15 \mathrm{~T}$ when samples are annealed around $700{ }^{\circ} \mathrm{C}$ in vacuum, as seen in Fig. 4(a) and it drops to almost half of that $(0.6 \mathrm{~T})$ when annealed at $800{ }^{\circ} \mathrm{C}$. On varying the annealing time from 5 to $120 \mathrm{~min}$ at $700{ }^{\circ} \mathrm{C}$, the coercivity increases monotonically up to $1.15, \mathrm{~T}$ after $30 \mathrm{~min}$, and then remains almost constant as shown in Fig. 4(b). The effect of changes in concentration of platinum p-salt is illustrated in Fig. 5. The composition of the deposits is directly affected by the bath composition, baths with higher concentration of $\mathrm{Pt}$ p-salt result in greater amounts of Pt in the deposits. In turn the changes in composition affect the coercivity of the de-

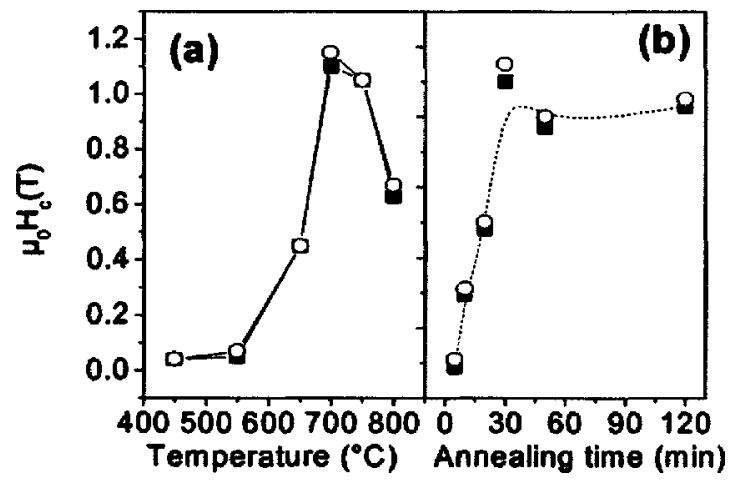

FIG. 4. Variation of the coercivity measured in the plane of the membrane (squares) and out of plane (circles) with (a) annealing temperature and (b) annealing time. 


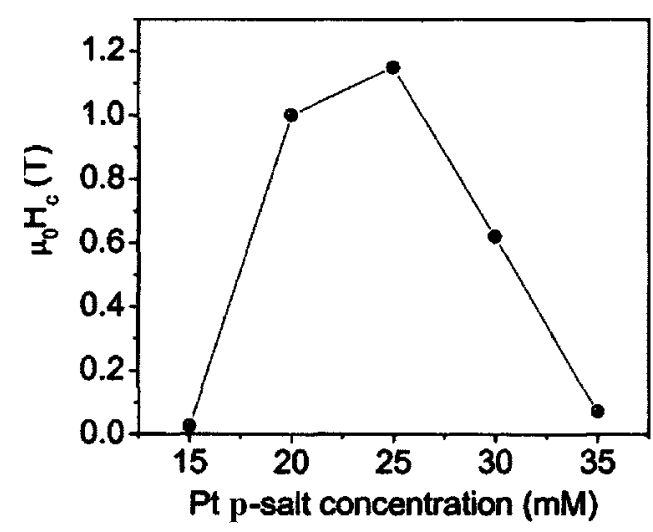

FIG. 5. Coercivity dependence on platinum p-salt concentration. Samples were annealed at $700{ }^{\circ} \mathrm{C}$ for $30 \mathrm{~min}$.

posit. As can be seen there is a window of concentration which leads to high coercivity. Some further improvement in coercivity, up to $1.3 \mathrm{~T}$, is obtained by annealing in hydrogen.

The behavior of the virgin curve shown in Fig. 3(b) is characteristics of a pinning-type magnet. Samples are magnetically isotropic and the saturation magnetization of the $\mathrm{CoPt}$ is estimated as $41 \mathrm{~J} \mathrm{~T}^{-1} \mathrm{~kg}^{-1}$. The isotropic behavior of the samples is associated with a random distribution of the $c$ axis within the nanowires, which is in line with the polycrystalline structure observed in the x-ray diffraction measurements. An anisotropic magnet would have been preferred, but our procedure did not lead to any crystalline orientation. Nevertheless, hysteresis loops are square, with $M_{r} / M_{s}$ of about 0.95 , which indicates a strong intergrain exchange coupling within the nanowires. Recoil curves were measured for samples annealed at different temperatures. First, the sample is brought to saturation followed by a measurement of the remanence at zero field. Then, a series of reverse fields in the opposite direction to the remanence is applied. Once the minimum value of field is reached on the recoil curve, the field is increased to zero and the recoil curve measured. The process is repeated cyclically for different points in the second quadrant. Data for samples annealed at $650{ }^{\circ} \mathrm{C}$, Fig. 3(c), show larger reversible magnetization than those for samples annealed at $700{ }^{\circ} \mathrm{C}$, Fig. 3(d). This is due to the fact that at $650{ }^{\circ} \mathrm{C}$ the transformation from disordered to ordered $L 1_{0}$ phase is far from complete. The soft disordered phase is stiffened by exchange coupling to the hard ordered phasethe exchange spring. ${ }^{20}$ As the annealing temperature is raised to $700{ }^{\circ} \mathrm{C}$, most of the disordered phase is converted to $L 1_{0}$ which shows a smaller reversible magnetization.

The electroplated structures were magnetized to create pole patterns giving a stray field with alternating polarity. The simplest is a structure consisting of stripes equally separated uniformly magnetized perpendicular to the plane, illustrated in Fig. 6(a).

The dependence of the coercivity of CoPt on the composition of the bath or the heat treatment can also be exploited to produce interdigitated stripes. Electrodes A [inset Fig. 6(b)] are first used to deposit the odd-numbered poles from a bath which leads to high coercivity and electrodes B are then used to electrodeposit the even-numbered poles from a bath which produce small coercivity. A high field is
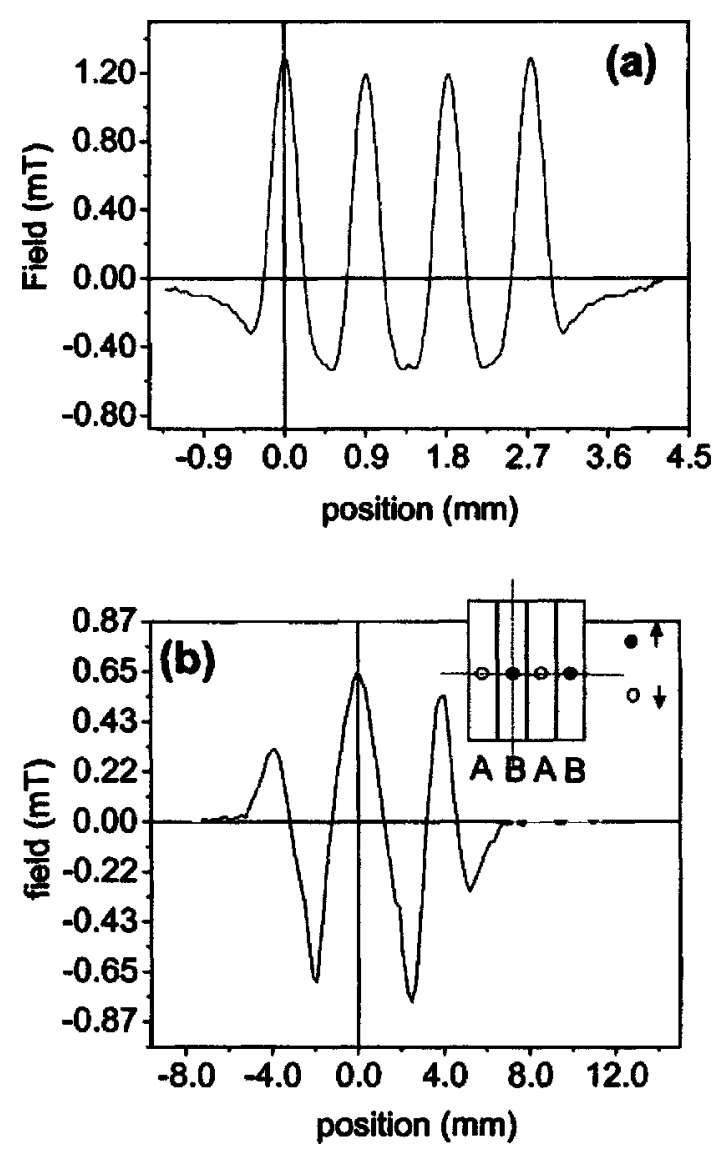

FIG. 6. (a) Electrodeposited CoPt stripes pattern equally spaced which produce an alternating stray field with a $450-\mu \mathrm{m}$ pole. (b) Inset electrode patterns for a multipole stripes and stray field contours on $z$ direction for a multipole magnet produced by a two-step electrodeposition process.

then applied to saturate the magnetization of both patterns A and $\mathrm{B}$, and the field is switched to a negative value greater than the coercivity of the softer B stripes, but less than that of the harder A electrodes $(1.15 \mathrm{~T})$ leading to a magnetization pattern of oppositely magnetized A and B stripes [Fig. 6(b)]. Alternatively two different thermal treatments may be used to generate different coercivities in the two sets of poles.

\section{CONCLUSION}

In conclusion, we have shown that electrodeposited $\mathrm{CoPt}$ alloys have a greatly improved loop shape and coercivity when they are deposited in nanoporous templates. They can be used to prepare microscale permanent magnets. The dependence of coercivity on composition or thermal treatment can be exploited in a two-step process which permits to magnetize oppositely neighboring regions with a fine pole pitch. The structured permanent magnets produced by template electrodeposition open the possibility of creating complex permanent magnet patterns on a microscale and realizing magnetic MEMS devices.

\section{ACKNOWLEDGMENTS}

This research was supported by the EU as part of the $\mathrm{M}^{2}$ EMS project in the ICT Program, and by Science Foundation Ireland. 
${ }^{1}$ O. Cugat, J. Delamare, and G. Reyne, IEEE Trans. Magn. 39, 3607 (2003).

${ }^{2} \mathrm{O}$. Cugat, Micro-actionneurs Electromagnetiques-MAGMAS (Hermes Science, Paris, 2002).

${ }^{3}$ S. A. Romero, D. R. Cornejo, F. M. Rhen, A. C. Neiva, M. H. Tabacniks, and F. P. Missell, J. Appl. Phys. 87, 6965 (2000).

${ }^{4}$ A. S. Lileev, A. A. Parilov, and V. G. Blatov, J. Magn. Magn. Mater. 242-245, 1300 (2002).

${ }^{5}$ M. Venkatesan, J. Buschbeck, F. M. F. Rhen, and J. M. D. Coey, J. Magn. Magn. Mater. 272-276, E881 (2004).

${ }^{6}$ X. Wang and G. R. Han, Microelectron. Eng. 66, 166 (2003).

${ }^{7}$ H. Ito, A. Taenaka, Y. Nagasaki, K. Kataoka, M. Kato, and T. Tsuruta, Polymer 37, 633 (1996).

${ }^{8}$ F. M. F. Rhen and J. M. D. Coey, J. Magn. Magn. Mater. 272-276, E883 (2004).

${ }^{9}$ I. Zana and G. Zangari, J. Magn. Magn. Mater. 272-276, 1698 (2004).

${ }^{10}$ G. Zangari, B. Bozzini, P. L. Cavalotti, G. Fontana, P. G. Maisto, and E. Terrenzio, J. Magn. Magn. Mater. 133, 511 (1994).

${ }^{11}$ F. M. F. Rhen, G. Hinds, C. O'Reilly, and J. M. D. Coey, IEEE Trans.
Magn. 39, 2699 (2003).

${ }^{12}$ L. Callegaro, E. Puppin, P. L. Cavalotti, and G. Zangari, J. Magn. Magn. Mater. 155, 190 (1996).

${ }^{13}$ J. Xu, X. Huang, G. Xie, Y. Fang, and D. Liu, Mater. Res. Bull. 39, 811 (2004).

${ }^{14}$ T. G. Sorop, K. Nielsch, P. Goring, M. Krol, W. Blau, R. B. Wehspohn, U. Gosele, and L. J. de Longh, J. Magn. Magn. Mater. 272-276, 1656 (2004).

${ }^{15}$ M. Kröll, W. J. Blau, D. Grandjean, R. E. Benfield, F. Luis, P. M. Paulus, and L. J. de Jongh, J. Magn. Magn. Mater. 249, 241 (2002).

${ }^{16}$ Y. C. Sui, R. Skomski, K. D. Sorge, and D. J. Sellmeyer, Appl. Phys. Lett. 84, 1525 (2004).

${ }^{17}$ J. Mallet, K. Yu-Zhang, C. L. Chien, T. S. Eagleton, and P. C. Searson, Appl. Phys. Lett. 84, 3900 (2004).

${ }^{18}$ N. Yasui, A. Imada, and T. Den, Appl. Phys. Lett. 83, 3347 (2003).

${ }^{19}$ G. Zangari, P. Bucher, N. Lecis, P. L. Cavalotti, L. Callegaro, and E. Puppin, J. Magn. Magn. Mater. 157-158, 256 (1996).

${ }^{20}$ E. F. Kneller and R. Hawig, IEEE Trans. Magn. 27, 3588 (1991). 